\title{
Die Natur des Fibrinferments.
}

\author{
Von
}

C. A. Pekelharing und W. Hniskamp.

(Der Redaktion zugegangen am 24. Mai 1903.)

Vor kurzer Zeit hat Hammarsten gegen die von uns verteidigte Meinung, daß sowohl das Nucleohiston als das andere Nucleoproteid des Thymusextraktes, mit Kalk verbunden, Fibrinferment bilden könne, einige Einwände erhoben. ${ }^{1}$ ) «Die Tatsache, daß verschiedenartige Proteide als Fibrinferment wirken können," sagt Hammarsten am Schluß seiner Bemerkungen, "erklärt sich auch nach meiner Ansicht am einfachsten durch die Annahme, daß kleine Mengen Thrombin oder Prothrombin in mehreren Organen sich vorfinden und von Proteiden - ebenso wie von Globulinen - bei ihrer Ausfällung, mit niedergerissen werden.»

Daß es augenblicklich nicht möglich ist, die Berechtigung dieser Auffassung - welche sich auch bei der Frage nach der Natur anderer Fermente immer wieder geltend macht endgültig zu widerlegen, wird von uns vollkommen zugegeben. Dennoch glauben wir, daß die Gründe, welche dafür sprechen, daß verschiedene Nucleoproteide selbst als Vorstufen von Fibrinferment $\mathrm{zu}$ betrachten seien, wichtiger sind als diejenigen, welche für die Meinung, das Ferment sei immer derselbe Stoff und in den Niederschlägen von Eiweißstoffen nur als Beimischung vorhanden, anzuführen sind.

Es ist wohl bemerkenswert, daß es eben phosphorhaltige Proteide sind, welche, aus Blut, aus Organextrakten, oder auch aus Milch, gefällt, die Wirkung von Fibrinferment zeigen. Zwar besitzt auch aus Blutserum gefälltes Globulin diese Wirkung,

1) Ergebnisse der Physiologie, 1. Jahrg., I, Biochemie S. 330. 
dasselbe ist dann aber auch immer mit Nucleoproteid verunreinigt. Wird verdünntes Blutserum mit Essigsäure neutralisiert, so bildet sich ein großer Niederschlag, hauptsächlich von Globulin, welcher eine viel schwächere Fermentwirkung hat, als die viel kleinere, hauptsächlich aus Nucleoproteid bestehende Fällung, welche durch nicht zu schwache Ansäuerung des Serums mit Essigsäure hervorgerufen wird. Wäre das Ferment ein nur mit dem Eiweißniederschlag mitgerissener Stoff, so dürfte man erwarten, daß der größere Niederschlag auch mehr davon enthalten würde.

Die Möglichkeit, daß unter sich verschiedene Nucleoproteide dennoch die gleiche Fermentwirkung haben könnten, wird von Hammarsten zugegeben. Er bemerkt aber, daß «es sehr auffallend wäre, wenn so wesentlich verschiedene Stoffe als Fibrinferment wirken könnten».

Als nicht weniger auffallend ist es aber, wie wir glauben, $\mathrm{zu}$ betrachten, daß nicht nur die beiden aus dem Thymusextrakt erhaltenen Nucleoproteide, sondern auch das aus dem Blutplasma bereitete Kalkverbindungen liefern, welche, bei Abwesenheit anderer Salze, am schwersten in $0,1 \% \mathrm{CaCl}_{2}$ enthaltenden Flïssigkeiten löslich sind und eben bei diesem Gehalt an Kalksalz am besten in Salz gelöstes Fibrinogen zur Gerinnung bringen. ${ }^{1}$ ) Wenn man dafür hält, das Zymogen sei irgend eine unbekannte Beimischung, so wäre man gezwungen, anzunehmen, daß diese eben in demselben Maß von Kalksalzen beeinflußt wird als die Nucleoproteide, womit sie zufälligerweise gemischt vorkommt. Außerdem hätte man anzunehmen, daß diese Beimischung von $\mathrm{MgSO}_{4}{ }^{2}$ ) und von $\mathrm{BaCl}_{2}{ }^{3}$ ) verhindert werden würde, mit Kalksalzen Ferment zu bilden, eben dann, wenn diese Salze in genügender Menge vorhanden sind, um dem Zustandekommen einer Kalkverbindung der Nucleoproteide vorzubeugen.

Ein anderer Einwand, welchen Hammarsten gegen unsere Auffassung erhebt, ist, daß in unseren Versuchen die Fermentwirkung des Thymusextraktes viel zu gering war, um dieselbe

1) Diese Zeitschr., Bd. XXXII., S. 187.

2) Centralbl. f. Physiol., 1895, S. 108.

$\left.{ }^{3}\right)$ Diese Zeitschr., Bd. XXXII, S. 195. 
den darin enthaltenen Nicluoproteiden zuschreiben zu können. Hier liegt aber ein Mißverständnis vor. In den von Ham marste $n$ citierten Versuchen wurde bezweckt, den Gehalt an Kalksalz, bei welchem die Gerinnung am besten stattfand, zu bestimmen - die Menge des Thymusextractes kam dabei nur insoweit in Betracht, als sie in allen Versuchen gleich groß und, unter übrigens günstigen Verhältnissen, sicher genügend sein mußte zur Hervorrufung der Gerinnung. Daß in diesen Versuchen die Gerinnung erst nach $1 \frac{1}{2}$ bis 2 Stunden vollständig war, kann nicht als Beweis gelten, daß das Extrakt verhältnismäßig zymogenarm war. Denn die Gerinnungsgeschwindigkeit hängt nicht nur von der Fermentmenge und von der Temperatur, sondern auch von verschiedenen, größtenteils unbekannten, Nebenumständen $a b$, von Beimischungen, welche die Fibrinausscheidung zu verzögern, oder sogar zu verhindern imstande sind. Wahrscheinlich spielen dabei auch die autolytischen Wirkungen eine Rolle. Nicht selten findet man, wenn im Plasma oder in einer reinen Fibrinogenlösung durch Zusatz von irgend einem Organextrakt oder von einer Caseinlösung (mit Hilfe von Kalk) Gerinnung hervorgerufen wird, daß das ausgeschiedene Fibrin nach einiger Zeit wieder gelöst wird; bisweilen fängt sogar die Wiederauflösung schon an, als sich noch nur eine sehr weiche Fibringallerte gebildet hat.

Während wir früher schon öfters gesehen hatten, daß die Thymusproteide auch in sehr winzigen Mengen Gerinnung hervorzurufen vermögen, haben wir jetzt, durch Hammarstens Bemerkungen angeregt, die Sache genauer untersucht.

$5 \mathrm{ccm}$ einer nach Hammarstens Methode aus Pferdeoder Rindsblut hergestellten Fibrinogenlösung wurde mit $1 \mathrm{ccm}$ einer Nucleoproteidlösung und 1 Tropfen $10 \%$ iger $\mathrm{CaCl}_{2}$-Lösung $\left(6 \mathrm{mg} \mathrm{CaCl}_{2}\right)$ vermischt. Die Nucleoproteidlösung wurde stufenweise verdünnt und dann wurde untersucht, bei welcher Verdünnung dieselbe noch imstande war, innerhalb etwa 12 Stunden Gerinnung hervorzurufen.

So wurden folgende Ergebnisse erhalten:

1. Eine Ca-Nucleohistonlösung, hergestellt durch Vermischen des Thymusextraktes mit $\mathrm{CaCl}_{2}$ bis zu einem Gehalt 
von $0,1 \%$, Abzentrifugieren des Niederschlages, Lösen mit Hilfe von sehr wenig Ammoniak in Wasser, Wiederausfällen mit $\mathrm{CaCl}_{2}$ und dann Auflösen in 3\%iger Kochsalzlösung, verursachte noch vollständige Gerinnung, als $1 \mathrm{ccm}$ derselben $0,47 \mathrm{mg}$ feste Substanz (aschefrei) enthielt. Das Gerinnungsgemisch $(6 \mathrm{ccm})$ enthielt also in $1 \mathrm{ccm} 0,08 \mathrm{mg}$ Nucleohiston.

2. Ca-Nucleohiston wurde durch $0,3 \%$ ige Essigsäure von Calcium befreit, zweimal mit Wasser ausgewaschen und dann mit Hilfe von Ammoniak in Wasser gelöst. Die so erhaltene, neutrale, nahezu salzfreie Nucleohistonlösung enthielt in $25 \mathrm{ccm}$ 0,1775 g Trockensubstanz. Von der zehnfach mit Wasser verdünnten Lösung verursachte $1 \mathrm{ccm}$, also $0,7 \mathrm{mg}$ Nucleohiston, noch vollständige Gerinnung. Auf $1 \mathrm{ccm}$ des Gerinnungsgemisches kam also 0,12 mg Nucleohiston.

3. Nucleoproteid, aus Thymusextrakt nach Entfernung des Nucleohistons mittels $\mathrm{CaCl}_{2}$ mit Essigsäure gefällt, mit Wasser ausgewaschen, dann mit Ammoniak bis zu neutraler Reaktion in Wasser gelöst. Die Lösung enthielt in $25 \mathrm{ccm} 0,1048 \mathrm{~g}$ Trockensubstanz. $1 \mathrm{ccm}$ der bis auf das vierfache Volumen mit Wasser verdünnten Lösung, 0,17 mg Nucleoproteid auf $1 \mathrm{ccm}$ Gerinnungsgemisch entsprechend, rief noch nahezu vollständige Gerinnung hervor, während $1 \mathrm{ccm}$ der fünfzigfach verdünnten Lösung, also 0,014 mg Nucleoproteid auf $1 \mathrm{ccm}$ Gerinnungsgemisch, noch die Entstehung eines, wenngleich weichen Koagulums veranlaßte.

4. Natriumnucleohiston wurde in folgender Weise bereitet: Aus Thymusextrakt mittels $\mathrm{CaCl}_{2}$ ausgefälltes Calciumnucleohiston wurde in Wasser mit sehr wenig Ammoniak gelöst, nochmals mit $\mathrm{CaCl}_{2}$ gefällt und in Wasser verteilt. In dieser Flüssigkeit wurde durch Kochsalzzusatz bis $\mathrm{zu}$ einem Gehalt von $0,8 \%$ ein Niederschlag, in welchem das Nucleohiston größtenteils an Natrium gebunden war, hervorgerufen und in $3 \%$ iger $\mathrm{NaCl}$ gelöst. Die Lösung enthielt in $20 \mathrm{ccm} 0,1297 \mathrm{~g}$ aschefreie Substanz. $1 \mathrm{ccm}$ der zwanzigfach mit $3 \%$ iger $\mathrm{NaCl}$ verdünnter Lösung verursachte vollständige Gerinnung, also bei einem Gehalt des Gerinnungsgemisches von $0,055 \mathrm{mg}$ Nucleohiston auf $1 \mathrm{ccm}$. 
5. Aus dem sub 4 erwähnten, von Nucleohiston befreitem Thymusextrakt wurde mittels Essigsäure Nucleoproteid bereitet. Der Niederschlag wurde mit Wasser ausgewaschen und dann mit Hilfe von Natronlauge bis zur äußerst schwachen alkalischen Reaktion in Wasser aufgelöst. Der Lösung wurde bis zu einem Gehalt von $3 \%$ Kochsalz zugesetzt. In $20 \mathrm{ccm}$ enthielt diese Lösung 0,1094 aschefreies Nucleoproteid. $1 \mathrm{ccm}$ der zwanzigfach mit $3 \%$ iger $\mathrm{NaCl}$ verdünnten Lösung, also $0,046 \mathrm{mg}$ Nucleoproteid auf $1 \mathrm{ccm}$ Gerinnungsgemisch, rief vollständige Gerinnung hervor.

Selbstverständlich wurde durch Kontrollversuche festgestellt, daß Zusatz von Chlorcalcium allein die Fibrinogenlösung nicht zum Gerinnen brachte.

Die von Hammarsten gebrauchten, sehr kräftig wirkenden Fibrinfermentlösungen ${ }^{1}$ ) enthielten 0,03 bis $0,04 \%$ Trockensubstanz. Hier wurde die Fermentlösung zu gleichen Teilen mit der Fibrinogenlösung vermischt, sodaß hier $1 \mathrm{ccm}$ des Gerinnungsgemisches 0,15 bis $0,2 \mathrm{mg}$ trockenes Ferment enthielt.

Die Mengen der Thymusnucleoproteide, welche zum Hervorrufen der Gerinnung erforderlich sind, sind also von derselben Ordnung als die Mengen des aus Blutserum bereiteten Fibrinferments, welche Gerinnung veranlassen können. Sicher sind diese Mengen nicht so groß, daß man darin einen Grund finden könnte für die Annahme, daß die Wirkung nicht auf den Nucleoproteiden selbst, sondern auf unbekannten Beimischungen beruhen sollte. Auch spricht die Wirksamkeit des anderen, neben dem Nucleohiston im Thymusextrakt vorhandenen Nucleoproteids gegen eine solche Annahme. Dieses Nucleoproteid wird ja erst aus dem Extrakt ausgefällt, nachdem das Nucleohiston mittels $\mathrm{CaCl}_{2}$ daraus entfernt worden ist. Wäre die Fermentwirkung an beim Ausfällen der Proteide mitgerissene Stoffe gebunden, so müßte man erwarten, daß der zuerst gebildete Niederschlag, das Nucleohiston, mehr davon enthalten würde als der zweite, welcher aus einer von jenen Beimischungen schon teilweise befreiten Lösung gefällt wird. Dennoch stellte es sich heraus, daß vom zweiten Nucleoproteid zum Hervorrufen der Gerinnung nicht mehr nötig war wie vom Nucleohiston.

1) Diese Zeitschr., Bd. XXVIII, S. 100. 
Eine andere Tatsache, welche wohl schwerlich mit der von Hammarsten bevorzugten Auffassung in Einklang $\mathrm{zu}$ bringen ist, wollen wir noch hervorheben, die Fähigkeit nämlich des Nucleohistons, auch dann mit Hilfe von Kalk Gerinnung zu verursachen, wenn es nicht in der gewöhnlichen Weise, sondern mittels Elektrolyse ausgefällt worden ist. ${ }^{1}$ ) In diesem Fall wird ja das Proteid in ionisiertem Zustand durch die Flüssigkeit geführt und erst ausgefällt, wenn es die Anode erreicht hat. Dann kann also von den beigemischten Stoffen nur soviel mitgerissen werden, als sich davon im U-Rohr in der unmittelbaren Nähe der Anode befindet. Man hätte also, wenn man erklären will, daß das Nucleohiston auch unter diesen Verhältnissen seine Fermentwirkung nicht verliert, die ganz unbegründete Annahme zur Hilfe zu rufen, daß jene unbekannten Beimischungen auch als Anionen vom Strom mitgeführt werden sollten.

Es gibt aber noch einen, unserer Meinung nach, wichtigeren Grund, welcher für die von uns verteidigte Auffassung spricht. Wenn das Ferment in irgend einer unbekannten Substanz zu suchen ist, welche von andersartigen Niederschlägen mechanisch mitgerissen wird, dann muß dieses Ferment die gleichen Eigenschaften besitzen, speziell auch bei der gleichen Temperatur seine Wirksamkeit verlieren, einerlei, ob es nun mit dem einen oder mit dem anderen Nucleoproteid gemischt vorkommt. Wenn dagegen die Nucleoproteide selbst, mit Hilfe von Kalksalzen, das Ferment bilden, so ist zu erwarten, daß verschiedene Nucleoproteide auch bei verschiedenen Temperaturen ihre Eigenschaft als Zymogen einbüßen werden.

Früher ist schon mitgeteilt worden, $\left.{ }^{2}\right)$ daß es in dieser Hinsicht tatsächlich einen Unterschied gibt. Es stellte sich heraus, daß das Nucleoproteid des Blutplasmas leichter durch Erhitzen der Fähigkeit, Ferment zu bilden, beraubt wird, als die aus Thymus und aus Testikeln erhaltenen Nucleoproteide und als das Casein der Kuhmilch. Dagegen kann aber eingewendet werden, daß Fibrinferment verschiedener Herkunft nicht immer

1) Diese Zeitschr., Bd. XXXIV, S. 35.

2) Untersuchungen über das Fibrinferment. Kon. Akad. v. Wetensch. Amsterdam, 1892, S. 28. 
genau dieselbe Substanz zu sein braucht, wenn man auch so große Unterschiede, wie zwischen verschiedenen Nucleoproteiden gefunden werden, nicht für wahrscheinlich hält.

Nimmt man aber an, daß die verschiedenen Nucleoproteide desselben Thymusextraktes die Fähigkeit, Ferment zu bilden, mechanisch mitgerissenen Beimischungen verdanken, dann ist man auch wohl genötigt, dafür zu halten, daß die dem einen Nucleoproteid anhaftenden Teilchen derselben Art sind, wie diejenigen, welche aus demselben Extrakt von dem anderen Nucleoproteid mitgerissen worden sind. Mit dieser Annahme ist nun folgende Beobachtung wohl kaum in Einklang zu bringen.

Aus Thymusextrakt wurde Nucleohiston mittels $\mathrm{CaCl}_{2}$ gefällt, durch noch einmal wiederholte Fällung mit $\mathrm{CaCl}_{2}$ gereinigt, in Wasser verteilt und durch Kochsalzzusatz bis zu einem Gehalt von $0,9 \%$ gefällt. Das so erhaltene Nucleohiston, welches zum weitaus größten Teil an Natrium gebunden war, wurde in $3 \%$ iger Kochsalzlösung aufgelöst.

Aus dem mit der Zentrifuge von Ca-Nucleohiston befreiten Extrakt wurde mittels Essigsäure das andere Nucleoproteid gefällt. Dasselbe wurde mit Wasser ausgewaschen, dann mit Ammoniak bis zu genau neutraler Reaktion in Wasser gelöst und bis zu einem Gehalt von 3\% mit Kochsalz versetzt.

Von jeder dieser Lösungen verursachte $1 \mathrm{ccm}$, mit $5 \mathrm{ccm}$ Fibrinogenlösung und $6 \mathrm{mg} \mathrm{CaCl}_{2}$ vermischt, vollständige $\mathrm{Ge}$ rinnung. Ein Teil jeder Lösung wurde 15 Minuten lang in demselben Wasserbad auf $54^{\circ} \mathrm{C}$. erhitzt. Danach verursachte $1 \mathrm{ccm}$ der Nucleohistonlösung in $5 \mathrm{ccm}$ Fibrinogen mit $6 \mathrm{mg} \mathrm{CaCl}_{2}$ noch ebenso vollständige Gerinnung wie vor der Erhitzung, während die Nucleoproteidlösung jetzt gar keine Gerinnung hervorrief.

Nach Erhitzung auf $60^{\circ}$ C., 15 Minuten lang, und Vermischung mit Fibrinogen und $\mathrm{CaCl}_{2}$ in demselben Verhältnis wie zuvor bildete sich im Röhrchen mit Nucleohiston noch ein weiches, durch Schütteln zu zerstückelndes Koagulum; im Röhrchen mit Nucleoproteid war, wie zu erwarten, keine Spur von Gerinnung zu beobachten. 
Nach Erhitzung auf $80^{\circ}$ C., 10 Minuten lang, hatten beide Lösungen das Vermögen, Gerinnung zu veranlassen, verloren.

Wiederholungen des Versuches mit den oben sub 4 und 5 erwähnten Lösungen, wobei dafür Sorge getragen war, daß das Nucleohiston und das Nucleoproteid in ungefähr der gleichen Konzentration vorhanden waren, ergab dasselbe.

Dieses Ergebnis scheint uns nicht wohl zu erklären zu sein, bei der Annahme, daß das Fibrinferment aus einem mechanisch mit den Proteidniederschlägen mitgerissenen Zymogen herstamme, vollkommen in Übereinstimmung aber mit der Auffassung, daß die Fermentwirkung in dem eigentümlichen Bau der Nucleoproteide ihren Grund hat.

In der soeben citierten Arbeit ist erwähnt, daß «auch die (Wirksamkeit des Nucleoalbumins ${ }^{1}$ ) aus Thymus verloren geht, wenn es einige Zeit hindurch (10 Minuten) auf $60^{\circ} \mathrm{C}$. erhitzt wird». Dieser Befund ist mit dem jetzt beschriebenen nicht in Widerspruch. Das Gemisch von Proteiden, damals Nucleoalbumin genannt, das Wooldridgesche "Gewebsfibrinogen", wurde aus dem Thymusextrakt durch Fällen mit Essigsäure und Lösen des Niederschlages in 0,6\% von ein wenig Soda bereitet. ${ }^{2}$ ) Die filtrierte Lösung enthält dann, wie wir jetzt wissen, nur wenig Nucleohiston, sondern hauptsächlich das andere Nucleoproteid. Dieses wird, wie aus dem jetzt mitgeteilten Versuch hervorgeht, schon durch Erhitzung auf $54^{\circ} \mathrm{C}$. unwirksam. Die geringe Menge des in jener Lösung vorhandenen Nucleohistons, welches durch Erhitzung auf $60^{\circ} \mathrm{C}$. während 15 Minuten zwar nicht völlig zerstört, aber doch in seiner Wirksamkeit sehr geschädigt wird, konnte in jenen Versuchen wohl kaum mehr in Betracht kommen.

Für die Erforschung des Einflusses der Erhitzung wurde Na-Nucleohiston gebraucht, und nicht Ca-Nucleohiston, da letzteres, in Kochsalz gelöst, schon bei Körpertemperatur allmählich verändert wird und in eine unlösliche Modifikation übergeht.

1) Die betreffende Arbeit wurde geschrieben, bevor Hammarsten die Unterscheidung zwischen Nucleoproteiden und Nucleoalbuminen sinführte.

2) Virchows Festschrift, 1891, I, S. 455. 
Eine einigermaßen scharfe Bestimmung der Gerinnungstemperatur der Thymusnucleoproteide war uns nicht möglich, weil beim Erhitzen eine flockige Ausscheidung nicht nur von der Geschwindigkeit und dem Grad der Temperaturerhöhung und von dem Salzgehalt der Lösung abhängt, sondern auch von der Natur der in der Lösung vorhandenen Salze. Daß aber die Thymusnucleoproteide in der Widerstandsfähigkeit gegen Erhitzen unter sich verschieden sind, ist wohl nicht $\mathrm{zu}$ bezweifeln.

Nach dem Angeführten glauben wir also dafürhalten zu dürfen, daß die Thymusnucleoproteide und gleichfalls das aus Blutplasma erhaltene selbst das Zymogen darstellen, aus welchem mit Hilfe von Kalksalzen das Fibrinferment entsteht. Während alle tatsächlichen Beobachtungen, soweit wir sehen können, mit dieser Auffassung im Einklang sind, ist man gezwungen, mehrere neue, ausschließlich zu diesem Zweck ersonnene und nicht von Beobachtungen gestützte Hypothesen zur Hilfe zu nehmen, wenn man die Fermentwirkung einem unbekannten, zufällig mit diesen Proteiden gemischten Stoff zuschreiben will.

Ist unsere Auffassung richtig, so liegt die Annahme auf der Hand, daß auch die aus anderen Organextrakten und aus Milch erhaltenen P-haltigen Eiweißstoffe, deren Fähigkeit, mit Kalk Fibrinogen gerinnen zu machen, bekannt ist, als Prothrombine zu betrachten seien. Die Kenntnis des Einflusses dieser Stoffe auf die Gerinnung ist aber noch zu lückenhaft, $\mathrm{da} ß$ es gestattet wäre, darüber anders als vermutungsweise zu sprechen.

Daß die Wirkung des Enzyms nicht, wie früher von einem von uns angenommen wurde, in einer Übertragung von Kalk besteht, infolge dessen sich das Fibrin als eine Kalkverbindung ausscheiden würde, ist von Hammarsten nachgewiesen und vom Autor der Hypothese nach eigener Prüfung vollkommen zugegeben worden. ${ }^{1}$ )

1) Proc. Kon. Akad. v. Wetensch., Amsterdam, 24. Nov. 1900. 